\title{
Meninas Catarinas: a vida perdida ao ser mãe
}

\author{
GIRLS FROM SANTA CATARINA: LOSING YOUR LIFE TO BECOME A MOTHER
}

\author{
NIÑAS DE SANTA CATARINA: LA VIDA PERDIDA AL SER MADRE
}

\section{Maria de Lourdes de Souza ${ }^{1}$, Diego Burgardt ${ }^{2}$, Luiz Alberto Peregrino Ferreira ${ }^{3}$, Maria Bettina Camargo Bub $^{4}$, Marisa Monticelli5 ${ }^{5}$ Haimée Emerich Lentz ${ }^{6}$}

\section{RESUMO}

A razão da mortalidade materna é um indicador para análise das condições de vida e de assistência prestada à mulher, e instrumento para avaliar o cumprimento dos direitos constitucionais relativos à vida e à saúde. Esta pesquisa demonstra a vida perdida de mulheres adolescentes associadas à gestação, parto e puerpéreo, em Santa Catarina, no período de 1994-2005. Tratase de um estudo exploratório quantitativo. Encontrou-se no período 72 mortes maternas em adolescentes, sendo que $67 \%$ foram obstétrica direta, $23 \%$ obstétrica indireta e $9 \%$ acidentais e incidentais. A razão da mortalidade materna entre adolescentes foi elevada e a maioria dos óbitos poderia ter sido evitada, pois existem recursos técnicos para prevenir e controlar suas causas. A situação identificada demonstra a ausência de assistência de qualidade. Para reduzir estas mortes é fundamental o cuidado de enfermagem, numa ação articulada com outros profissionais e diferentes segmentos organizados da sociedade.

\section{DESCRITORES}

Adolescente.

Mortalidade materna.

Cuidados de enfermagem.

\begin{abstract}
The Maternal Mortality Ratio (MMR) is an indicator that analyses the life conditions and care given to pregnant women, and indicates whether the constitutional rights related to life and health are being complied with. This study shows losses of lives among adolescent women associated with pregnancy, childbirth and the puerperal period, in the state of Santa Catarina between 1994 and 2005. It is an exploratory study of a quantitative nature. There were 72 maternal deaths in adolescents, $67 \%$ had a direct obstetric cause, $23 \%$ had an indirect obstetric cause and about $9 \%$ were classified as accidental or incidental. The Maternal Mortality Ratio in adolescents was high and most could have been prevented and its causes controlled. The identified situation shows an absence of quality health care. To reduce these deaths it is central that nursing action be combined with other professionals and other organized sectors of society.
\end{abstract}

\section{KEY WORDS}

Adolescent.

Maternal mortality.

Nursing care.

\section{RESUMEN}

La Tasa de Mortalidad Materna (MMR, según siglas en inglés) es un indicador para el análisis de las condiciones de vida y cuidados prestados a la mujer, y sirve como instrumento para evaluar el cumplimiento de los derechos constitucionales relacionados con la vida y la salud. Esta investigación analiza las defunciones de mujeres adolescentes por causas asociadas a la gestación, parto y puerperio, en el Estado de Santa Catarina (Brasil) durante el período 1994-2005. Se trata de un estudio exploratorio de naturaleza cuantitativa. Se detectaron en dicho periodo 72 muertes maternas en adolescentes, siendo $67 \%$ del tipo obstétricas directas, $23 \%$ obstétricas indirectas y $9 \%$ accidentales e incidentales. La Tasa de Mortalidad Materna entre adolescentes presentó valores elevados y la mayoría de las muertes podría haber sido evitada, siendo que existen recursos técnicos para prevenir y controlar las causas determinadas. Para reducir estas muertes, la atención de enfermería es fundamental, en acciones coordinadas con otros profesionales y diferentes segmentos organizados de la sociedad.

\section{DESCRIPTORES}

Adolescente.

Mortalidad materna.

Atención de enfermería.

${ }^{1}$ Enfermeira. Doutora em Saúde Pública pela Faculdade de Saúde Pública da Universidade de São Paulo. Docente do Programa de Pós-Graduação em Enfermagem da Universidade Federal de Santa Catarina. Membro do Grupo de Pesquisa Cuidando e Confortando. Vice-presidente do Comitê de Mortalidade Materna do Estado de Santa Catarina. Coordenadora Geral da Rede de Promoção ao Desenvolvimento da Enfermagem. Florianópolis, SC, Brasil. lourdesr@repensul.ufsc.br, repensul@uol.com.br ${ }^{2}$ Médico. Graduado pela Universidade Federal de Santa Catarina. Ex-Bolsista do Projeto Mortalidade de Adolescentes junto ao Comitê de Mortalidade Materna do Estado de Santa Catarina. Florianópolis, SC, Brasil. diego_burgardt@yahoo.com.br ${ }^{3}$ Professor do Departamento de Análises Clínicas da Universidade Federal de Santa Catarina. Doutorando do Programa de Pós-Graduação da Universidade Federal de Santa Catarina. Florianópolis, SC, Brasil. lulaperegrino@yahoo.com.br ${ }^{4}$ Enfermeira. Mestrado e Doutorado em Enfermagem pela Universidade Federal de Santa Catarina. Doutorado Sanduíche pela Universidade de Linköping, Suécia. Docente e Coordenadora de Intercâmbios e Convênios Internacionais do Programa de Pós Graduação em Enfermagem da Universidade Federal de Santa Catarina. Líder do Núcleo de Estudos em Filosofia e Saúde. Florianópolis, SC, Brasil. vito@unetsul.com.br ${ }^{5}$ Enfermeira. Doutora em Enfermagem. Docente do Departamento de Enfermagem e do Programa de Pós Graduação em Enfermagem da Universidade Federal de Santa Catarina. Vice-líder do Grupo de Pesquisa em Enfermagem na Saúde da Mulher e do Recém-nascido. Florianópolis, SC, Brasil. marisa@nfr.ufsc.br ${ }^{6}$ Enfermeira. Mestre em Saúde Pública. Presidente do Comitê de Mortalidade Materna do Estado de Santa Catarina. Florianópolis, SC, Brasil. haimeelentz@hotmail.com

$210 \begin{aligned} & \text { Rev Esc Enferm USP } \text { Recebido: 03/07/2008 } \\ & \text { 2010; 44(2):318-23 } \text { Aprovado: 29/04/2009 } \\ & \text { www.ee.usp.br/reeuspl } \text { Português/Inglês } \\ & \text { www.scielo.br/reeusp }\end{aligned}$




\section{INTRODUÇÃO}

Os indicadores de saúde são instrumentos de avaliação com os quais se pode obter dados sobre atributos e dimensões da saúde de uma dada população.

A razão da mortalidade materna (RMM) é um indicador cuja análise revela o acesso da mulher à assistência à saúde, os riscos da gestação e do parto, e a adequação do sistema de assistência à saúde em responder às suas necessidades. Isto significa dizer que demonstra a condição social e econômica da mulher ${ }^{(1)}$. A RMM é obtida pela divisão entre o número de mortes maternas e o número de nascidos vivos em determinado período, multiplicado por $100.000^{(1)}$.

O número de mulheres grávidas seria idealmente o denominador da RMM. Devido a insuficiência de dados sobre abortos e nascidos mortos, adota-se o número de nascidos vivos (NV), constante de sistemas de registros vitais, como uma aproximação ao número de mulheres grávidas.

Para se determinar a RMM é necessária a clareza de duas definições, a de morte materna e a de nascido vivo. Na primeira, de acordo com a décima revisão da Classificação Internacional de Doenças (CID-10), morte materna é a morte de uma mulher durante a gestação ou puerpéreo (período de 42 dias após o parto), independente da duração, da localização da gravidez, ou da causa relacionada ou agravada pela gestação ou por medidas tomadas em relação a ela, excetuando-se causas acidentais ou incidentais ${ }^{(2-3)}$.

As mortes maternas podem ser classificadas em obstétricas diretas e indiretas ${ }^{(3)}$. As obstétricas diretas resultam de complicações obstétricas da gestação, parto ou puerpéreo, intervenções, omissões, tratamentos incorretos ou de uma cadeia de eventos originada em uma das situações anteriores e representam a principal causa de morte materna nos países em desenvolvimento. São exemplos: hemorragia, infecção puerperal e hipertensão(3). As obstétricas indiretas decorrem de doenças pré-existentes ou surgidas na gravidez, mas agravadas durante a gestação. Por exemplo, cardiopatias, colagenoses, diabetes, neoplasias hormônio-dependentes e outras doenças crônicas ${ }^{(3)}$.

As causas acidentais (acidente de trânsito e homicídio) e incidentais (apendicite aguda ocorrendo na gravidez) constituem um grupo de mortes denominadas não-obstétricas ${ }^{(3)}$.

A Federação Internacional de Ginecologia e Obstetrícia (FIGO) recomenda que sejam consideradas como mortes maternas todas as mortes, independentemente da causa, que ocorram até 42 dias após o término da gestação e todas as mortes ocorridas até um ano após o parto, na qual tenha sido possível identificar como desencadeante o processo gestacional.

A segunda definição é a de nascido vivo (NV):

[...] é a expulsão ou extração completa do interior do corpo da mãe, independentemente da duração da gravidez, de um produto de concepção que, depois da separação, respire ou apresente qualquer outro sinal de vida, tal como batimentos do coração, pulsações do cordão umbilical ou movimentos efetivos dos músculos de contração voluntária, estando ou não cortado o cordão umbilical, e estando ou não desprendida a placenta. Cada produto de um nascimento que reúna essas condições se considera como uma criança viva ${ }^{(2)}$

A RMM deveria ser mais próxima de zero; isto indicaria que a gestação - um fenômeno previsível e controlado, que só ocorre em mulheres, e que tem duração prevista e suporte de tecnologia em diversas especialidades médicas estaria de fato recebendo a assistência devida. Não se pode esquecer, entretanto, as causas inevitáveis de óbitos e todo processo social com o qual convivem as mulheres (condições de vida, acesso e qualidade dos serviços de saúde), que interferem na RMM.

A Organização Mundial de Saúde (OMS) estimou para o ano 2000 a ocorrência de 529.000 mortes maternas em todo o mundo e que cerca de uma mulher morreria por minuto, durante o ciclo gravídico-puerperal. No Brasil foi estimada a ocorrência de 8.700 mortes maternas ${ }^{(1)}$.

Na realidade brasileira, a morte materna em adolescentes, mulheres de 10 a 19 anos, espelha as desigualdades sociais do país, como o pouco valor conferido à mulher. Além disto, para cada morte ocorrida, várias outras situações de risco de morte, com possíveis seqüelas permanentes, são totalmente desconhecidas.

Os contextos, internacional e nacional, impulsionaram o governo brasileiro a realizar esforços para cumprir os compromissos de proteger a saúde das mulheres e os direitos humanos. Um exemplo disso é o Pacto Nacional de Redução da Morte Materna e Neonatal, de $2004^{(4)}$.

O panorama apresentado justifica que estudos sobre mortalidade materna sejam realizados, com a finalidade de sua redução.

\section{OBJETIVO}

Demonstrar a morte de mulheres adolescentes associadas à gestação, parto e puerpéreo, no Estado de Santa Catarina, no período de 1994-2005.

\section{MÉTODO}

Trata-se de um estudo exploratório de natureza quantitativa, numa série histórica de dez anos, 1994 a 2005. Refere-se às mortes de mulheres adolescentes, registradas nas diferentes regiões do estado de Santa Catarina. As regiões foram agrupadas conforme é apresentado no Programa das Nações Unidas para o Desenvolvimento (PNUD): Norte, Vale do Itajaí, Grande Florianópolis, Sul, Planalto Serrano e Oeste. Cada região foi dividida em suas respectivas microrregiões e, nestas, identificados os municípios que as integram ${ }^{(5)}$. 
Posteriormente, foram coletados dados nos seguintes sistemas: Instituto Brasileiro de Geografia e Estatística $(\text { IBGE })^{(6)}$; Sistema de Informações sobre Mortalidade (SIM) ${ }^{(7)}$; Sistema de Informações de Nascidos Vivos (SINASC) ${ }^{(8)}$; Fichas de Investigação do Comitê de Mortalidade Materna do Estado de Santa Catarina - Secretaria de Estado da Saúde.

Uma vez realizadas as explorações quantitativas, os dados foram agrupados, segundo a causa de óbito. Para este agrupamento adotou-se a nona CID, na análise dos dados de 1994 a 1996 e, para os demais anos, a décima edição da $\mathrm{CID}^{(2)}$. Para a sistematização dos dados adotou-se planilha eletrônica produzida para este fim. Para cada Região, em um mesmo período, a razão de mortalidade materna (RMM) foi calculada pela divisão entre o número de mortes maternas, o número de nascidos vivos, multiplicado por 100.000 .

As causas das mortes maternas foram classificadas em obstétricas diretas (hemorragia, infecção, toxemia ou outros), obstétricas indiretas, acidentais ou incidentais. Previamente o projeto foi apresentado ao Comitê de Ética da Universidade Federal de Santa Catarina, em observância ao que determina a Resolução no 196/1996, do Ministério da Saúde ${ }^{(9)}$, e aprovado sob número 213/2005 e também foi aprovado e financiado pela FAPESC, sob Edital no 03/ 2004, Contrato no 14794/2005-0.

\section{RESULTADOS}

No estado de Santa Catarina, no ano de 2005, havia uma população estimada de 5.866.590. Desta, 2.944.728 era população feminina e 571.625 era de adolescentes, com idades entre 10 a 19 anos. A Tabela 1 mostra a distribuição da população feminina adolescente por região e a sua distribuição percentual. Proporcionalmente, por região, os percentuais variaram de 19,96 na Região Sul a 18,84\% na Região do Vale do Itajaí.

Tabela 1 - População feminina adolescente por região do Estado de Santa Catarina, no ano de 2005

\begin{tabular}{lccc}
\hline \multirow{2}{*}{ Regiões } & \multicolumn{2}{c}{ Adolescentes } & $\begin{array}{c}\text { População } \\
\text { feminina }\end{array}$ \\
\cline { 2 - 4 } & $\mathbf{N}$ & $\mathbf{\%}$ & $\mathbf{N}$ \\
\hline Grande Florianópolis & 87.892 & $18,85 \%$ & 466.358 \\
Norte & 111.924 & $19,61 \%$ & 570.671 \\
Oeste & 113.862 & $19,76 \%$ & 576.302 \\
Serrana & 41.589 & $19,86 \%$ & 209.449 \\
Sul & 89.223 & $19,96 \%$ & 447.045 \\
Vale de Itajaí & 127.135 & $18,84 \%$ & 674.903 \\
\hline Total & $\mathbf{5 7 1 . 6 2 5}$ & $\mathbf{1 9 , 4 1 \%}$ & $\mathbf{2 . 9 4 4 . 7 2 8}$ \\
\hline
\end{tabular}

No ano de 2005, no estado de Santa Catarina, ocorreram 15.616 registros de nascidos vivos, filhos de mães ado- lescentes, que corresponde a $18,47 \%$ do total de registros. Proporcionalmente, por região, os percentuais variaram de $24,90 \%$ na Região Serrana a 15,71\% na Região do Vale do Itajaí (Tabela 2).

Tabela 2 - Nascidos vivos, filhos de mulheres adolescentes por região do Estado de Santa Catarina, no ano de 2005

\begin{tabular}{lccc}
\hline \multirow{2}{*}{ Regióes } & \multicolumn{2}{c}{ Adolescentes } & Total \\
\cline { 2 - 4 } & $\mathbf{N}$ & $\mathbf{\%}$ & $\mathbf{N}$ \\
\hline Grande Florianópolis & 1.994 & $16,33 \%$ & 12.210 \\
Norte & 3.242 & $18,13 \%$ & 17.884 \\
Oeste & 3.486 & $20,83 \%$ & 16.732 \\
Serrana & 1.610 & $24,90 \%$ & 6.466 \\
Sul & 2.264 & $18,84 \%$ & 12.019 \\
Vale de Itajaí & 3.020 & $15,71 \%$ & 19.227 \\
\hline Total & $\mathbf{1 5 . 6 1 6}$ & $\mathbf{1 8 , 4 7 \%}$ & $\mathbf{8 4 . 5 3 8}$ \\
\hline
\end{tabular}

No período de 1994 a 2005 ocorreram 72 mortes maternas em adolescentes. Na Figura 1, mostra-se que o maior percentual de mortes maternas em adolescentes foi na Região Serrana, com $26,4 \%$ dos casos, e o menor foi de $3,4 \%$ na Região da Grande Florianópolis.

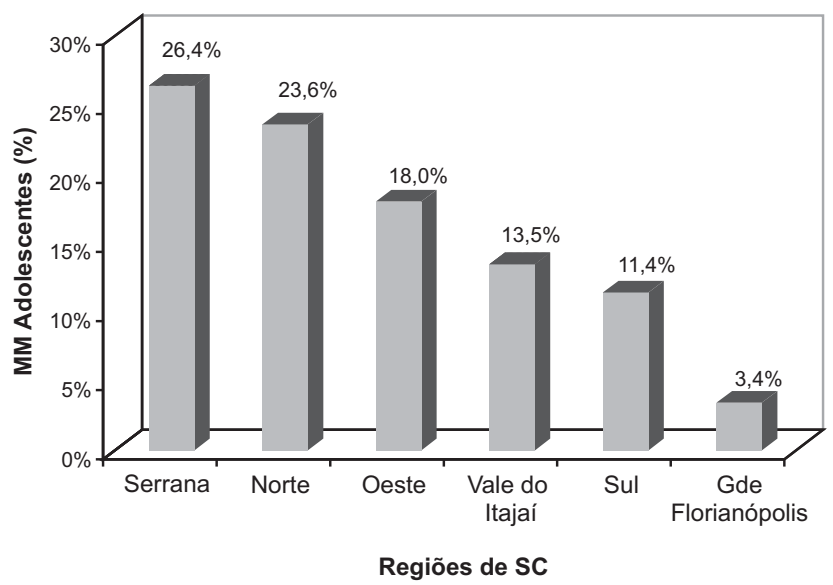

Figura 1 - Percentual de mortes maternas em adolescentes por região, no Estado de Santa Catarina, no período de 1994 a 2005

Na Tabela 3 apresentam-se as mortes maternas em adolescentes, por tipo de causa, ocorridas no estado de Santa Catarina, no período de 1996 a 2005. Das 64 mortes maternas registradas neste período, $67,18 \%$ foram obstétrica direta, 23,43\% obstétrica indireta, e 9,37\% acidentais e incidentais.

Dessas mortes maternas em adolescentes apresentadas na Tabela 3, as causas foram, em sua maioria, relacionadas ao parto e ao puerpéreo. 
Tabela 3 - Mortes maternas em adolescentes por tipo de causa, nas regiões do Estado de Santa Catarina, no período de 1996 a 2005

\begin{tabular}{|c|c|c|c|c|c|c|c|c|}
\hline \multirow{2}{*}{ Tipo de causa } & \multirow{2}{*}{$\begin{array}{c}\text { Grande } \\
\text { Florianópolis }\end{array}$} & \multirow{2}{*}{ Norte } & \multirow{2}{*}{ Oeste } & \multirow{2}{*}{$\begin{array}{l}\text { Região } \\
\text { Serrana }\end{array}$} & \multirow{2}{*}{ Sul } & \multirow{2}{*}{$\begin{array}{l}\text { Vale do } \\
\text { Itajaí }\end{array}$} & \multicolumn{2}{|c|}{ Total } \\
\hline & & & & & & & $\mathbf{N}$ & $\%$ \\
\hline Obstétrica direta & 1 & 12 & 10 & 10 & 5 & 5 & 43 & $(67,18)$ \\
\hline Hemorragia & 0 & 1 & 1 & 3 & 2 & 2 & 9 & $(14,06)$ \\
\hline Toxemia & 1 & 6 & 4 & 4 & 0 & 2 & 17 & $(26,56)$ \\
\hline Infecção & 0 & 4 & 3 & 1 & 2 & 0 & 10 & $(15,62)$ \\
\hline Outras causas diretas & 0 & 1 & 2 & 2 & 1 & 1 & 7 & $(10,93)$ \\
\hline Obstétrica indireta & $\mathbf{0}$ & 2 & 6 & $\mathbf{0}$ & 2 & 5 & 15 & $(23,43)$ \\
\hline Não relacionada & $\mathbf{0}$ & 2 & 2 & 1 & 1 & $\mathbf{0}$ & 6 & $(9,37)$ \\
\hline Total & 1 & 16 & 18 & 11 & 8 & 10 & 64 & \\
\hline
\end{tabular}

A RMM em adolescentes, por regiões do estado de Santa Catarina, no período de 1994 a 2005, é apresentada na Tabela 4. A Região Serrana, nos anos de 1994, 1995, 1996,

1997 e 2004 apresentou maior RMM, em relação às demais regiões. No período analisado a Região da Grande Florianópolis foi quem apresentou a menor RMM.

Tabela 4 - Razão de Mortalidade Materna em adolescentes, por regiões do Estado de Santa Catarina, no período de 1996 a 2005

\begin{tabular}{|c|c|c|c|c|c|c|c|}
\hline $\begin{array}{c}\text { Regiões } \\
\text { Ano }\end{array}$ & $\begin{array}{c}\text { Grande } \\
\text { Florianópolis }\end{array}$ & $\begin{array}{l}\text { Vale de } \\
\text { Itajaí }\end{array}$ & Norte & Oeste & $\begin{array}{c}\text { Região } \\
\text { Serrana }\end{array}$ & Sul & Total \\
\hline 1994 & 0,00 & 38,54 & 0,00 & 29,10 & 57,50 & 0,00 & 15,24 \\
\hline 1995 & 0,00 & 27,09 & 25,46 & 21,17 & 98,04 & 0,00 & 27,67 \\
\hline 1996 & 0,00 & 0,00 & 25,00 & 20,25 & 94,47 & 35,42 & 29,33 \\
\hline 1997 & 0,00 & 26,41 & 47,03 & 43,09 & 93,41 & 35,04 & 50,18 \\
\hline 1998 & 0,00 & 0,00 & 50,24 & 83,95 & 0,00 & 68,56 & 49,73 \\
\hline 1999 & 0,00 & 0,00 & 96,88 & 45,31 & 0,00 & 0,00 & 38,42 \\
\hline 2000 & 0,00 & 26,41 & 0,00 & 21,69 & 52,38 & 69,98 & 25,41 \\
\hline 2001 & 0,00 & 28,71 & 56,10 & 48,54 & 53,56 & 37,33 & 38,74 \\
\hline 2002 & 0,00 & 60,48 & 30,72 & 51,64 & 56,85 & 0,00 & 35,19 \\
\hline 2003 & 0,00 & 95,82 & 32,71 & 0,00 & 59,74 & 41,53 & 37,63 \\
\hline 2004 & 48,38 & 63,01 & 62,74 & 83,19 & 169,59 & 0,00 & 68,38 \\
\hline 2005 & 0,00 & 0,00 & 30,85 & 28,69 & 0,00 & 0,00 & 12,81 \\
\hline Total & 3,64 & 29,07 & 39,39 & 39,88 & 61,42 & 25,24 & 35,14 \\
\hline
\end{tabular}

Na Tabela 4 pode-se observar a irregularidade dos valores da RMM, nas diferentes regiões. A Região Serrana apresentou os maiores valores e maior irregularidade. $\mathrm{Na}$ Região da Grande Florianópolis foi encontrada tendência regular da RMM, exceto para o ano de 2004 e nas demais regiões a tendência foi de irregularidade em todo o período analisado.

\section{DISCUSSÃO}

É diferente a magnitude de nascidos vivos, filhos de mães adolescentes, entre as regiões catarinenses, apesar do número semelhante de mulheres adolescentes em cada região. Os percentuais dos nascidos vivos filhos de mães adolescentes encontrados em Santa Catarina, particular- mente na Região Serrana (24,90\%), se aproximam aos identificados no Brasil em 2001, que eram de $23,3 \%^{(7-8)}$.

Em Santa Catarina, no ano de 2005, o percentual do total de registros de NV foi de $18,47 \%$ e se aproxima de achados encontrados em outras unidades da Federação, bem como no exterior, muito embora em nosso estudo não tenha sido computada a totalidade dos partos, ou seja, os natimortos não foram incluídos. No município de Ribeirão Preto (SP), no período de 1992 a 1996, um estudo mostrou que $16,6 \%$ dos partos ocorreram em adolescentes ${ }^{(10)}$. Em Minas Gerais, no ano de 2001, a proporção estimada de NV de mães adolescentes foi de $21,5 \%^{(11)}$. No ano de 2000 , o percentual de NV, filhos de mães adolescentes, foi de $22 \%$ nos Estados Unidos, 15\% na Inglaterra, 11\% no Canadá, 6\% na França, e $4 \%$ na Suécia ${ }^{(12)}$. 
O percentual de mortes maternas em adolescentes do estado de Santa Catarina, em estudo que analisou dados do período de 1980 a 1998 , foi de $13,3^{(13)}$. No presente estudo as diferenças percentuais observadas nas mortes maternas de adolescentes em todas as regiões podem indicar diferenças na qualidade dos serviços de saúde existentes. O maior percentual de mortes está na Região Serrana, na qual o risco de morte de adolescentes associado à gestação, parto e puerpéreo é oito vezes maior do que na Região da Grande Florianópolis, onde fica a capital do Estado. Esta informação confirma a importância da RMM como indicador da qualidade da assistência à saúde da população(1).

As irregularidades dos valores da RMM encontradas nas regiões de Santa Catarina indicam a ineficiência dos serviços de saúde em assistir as adolescentes. Não existe um sistema de seguimento da gestação das adolescentes, sendo que o sistema de referência e contra-referência não identifica o risco da gestação, parto e puerpéreo em adolescentes, por falta de planejamento e o abandono de mulheres gestantes nesta idade.

Não podemos esquecer que o Estatuto da Criança e Adolescente e a Constituição Federal determinam a responsabilidade do Estado em assegurar o direito à vida e à saúde com absoluta prioridade. Portanto, a morte materna em adolescentes indica também a falta do cumprimento desta responsabilidade ${ }^{(14)}$.

Os dados de RMM no presente estudo são semelhantes aos outros encontrados nas capitais brasileiras, variando de 42 por 100000 NV na região sul, a 73,2 por 100000 NV no Nordeste $^{(15)}$, com um valor nacional de 54.3 por 100000 NV.

As causas encontradas de mortes maternas em adolescentes, nas regiões de Santa Catarina, no período de 1996 a 2005 foram, em sua maioria, associadas às complicações obstétricas da gestação, parto ou puerpéreo, indicando mais uma vez as deficiências dos serviços de saúde. Cerca de $90 \%$ destas mortes são evitáveis com medidas simples, como um adequado pré-natal para a prevenção da toxemia gravídica, ou atuando de maneira preventiva no caso da infecção e hemorragia puerperal| ${ }^{(3,16)}$.

Os dados deste estudo foram semelhantes a outros, como os da cidade de Campinas (SP), com 85,5\% de mortes maternas do tipo obstétrica direta ${ }^{(17)}$. Outro trabalho sobre morte materna nas capitais brasileiras relatou um valor de $67,7 \%^{(15)}$

Os estudos da realidade brasileira sobre a mortalidade de adolescentes podem contribuir para a redução do impacto de um problema com esta magnitude. O registro dos dados, as análises e as inferências decorrentes são informações que possibilitam a definição de políticas de saúde pública, atendendo às necessidades da população.

Neste contexto, o cuidado de enfermagem deve ser incorporado na estratégia para redução da mortalidade em adolescentes, com ações na área de assistência, no regis- tro de dados, na investigação dos óbitos, na coleta e interpretação de dados, bem como na proposição de políticas assistenciais e de educação( ${ }^{(16)}$.

No que se refere às meninas catarinas que perdem a vida no desejo de ser mãe, muito se tem a aprender. Isto porque, a menina não nasce mulher; se torna mulher ${ }^{(18)}$. É sabido que uma das pontes para tornar-se mulher diante da sociedade é a maternidade. Assim, as meninas passam a ser reconhecidas como mulheres, na família ou na sociedade em geral. A menina demonstra que o desejo aflora e ele se sobrepõe à razão, inclusive às normas sociais, religiosas, familiares e dos serviços de saúde.

Analisar a vida perdida de mulheres adolescentes e suas causas associadas à gestação, parto e puerpéreo, no estado de Santa Catarina, no período de 1994 a 2005, é um grito de alerta para que os profissionais de saúde aprendam a aliarse entre si e com a sociedade, num exercício em que o conhecimento se transforme em sabedoria para viver a vida. A adolescente não deve perder a vida quando oferece à sociedade outra vida. A menina tem o direito de viver ao tornarse mulher-mãe, adulta, mesmo que dependente social. A legislação brasileira determina que o poder público deve assegurar, com absoluta prioridade, a efetivação dos direitos referentes à vida e à saúde, dentre outros ${ }^{(4,14)}$.

Portanto, os profissionais de saúde precisam também contribuir para o cumprimento da legislação, aprender a aprender sobre a vida em sociedade e a enfermagem, em particular, a demonstrar todos os dias à importância do cuidado de enfermagem na dualidade inexorável do ser humano - viver e morrer.

\section{CONCLUSÃO}

As informações apresentadas no presente trabalho são suficientes para orientar a definição de políticas públicas voltadas aos adolescentes e devem ser utilizadas para validar ações de planejamento e de educação, com vistas a reduzir os riscos de mortalidade em adolescentes associadas à gestação, parto ou puerpéreo.

As instituições de saúde, na esfera nacional, estadual ou municipal, devem atuar de modo conjunto e articulado, como uma rede, onde os programas sejam integrados. É o que deveria acontecer, por exemplo, com o Programa de Saúde da Família, o Programa de Saúde da Mulher e o Programa de Saúde do Adolescente.

As políticas deverão definir a atuação conjunta entre os profissionais de saúde, como forma de possibilitar a necessária integração e o trabalho em equipe. Necessitam também definir os mecanismos de educação permanente para que os profissionais aprendam a trabalhar em equipe e também registrem adequadamente os fatos e situações vivenciadas sob suas responsabilidades.

Na prática, a gravidez na adolescência requer assistência pré-natal diferenciada, com acompanhamento domici- 
liar pela enfermagem e inserção precoce no programa, com adoção de um protocolo de risco, de modo a viabilizar um término da gestação satisfatório, ou seja, com resultados que garantam a vida da mulher e do filho.

A enfermagem pode contribuir para a redução da maioria das mortes maternas em adolescentes realizando, por exemplo, a busca ativa de gestantes adolescentes para o pré-natal e o cuidado de enfermagem no domicílio. Além disso, o cuidado de enfermagem é fundamental no parto, especialmente nas primeiras setenta e duas horas pós-parto, seja ele via vaginal ou cesárea.

\section{REFERÊNCIAS}

1. Word Health Organization (WHO). Maternal mortality in 2000: estimates developed by WHO, UNICEF and UNFPA. Geneva; 2004.

2. Organização Mundial da Saúde (OMS). Classificação Estatística Internacional de Doenças e Problemas Relacionados à Saúde. 10a rev. São Paulo: Centro Colaborador da OMS para Classificação de Doenças em Português/Universidade de São Paulo; 1994. 2 v.

3. Souza ML, Laurenti R. Mortalidade materna: conceitos e aspectos estatísticos. São Paulo: Centro Colaborado da OMS para a Classificação das Doenças em Português; 1987. (Série Divulgação, n. 3).

4. Brasil. Ministério da Saúde. Portaria n. 427, de 22 de março de 2005. Institui a Comissão Nacional de Monitoramento e Avaliação da Implementação do Pacto Nacional pela Redução da Mortalidade Materna e Neonatal e dá outras providências [legislação na Internet]. Brasília; 2005. [citado 2007 out. 17]. Disponível em: http://dtr2001.saude.gov.br/sas/PORTARIAS/ Port2005/GM/GM-427.htm

5. Programa das Nações Unidas para o Desenvolvimento (PNUD). Atlas do Desenvolvimento Humano [texto na Internet]. 2007 [citado 2007 set. 20]. Disponível em: http://www.pnud.org.br/ atlas/

6. Instituto Brasileiro de Geografia e Estatística (IBGE) [homepage na Internet]. Rio de Janeiro; 2007 [citado 2007 set. 25]. Disponível em: http://www.ibge.gov.br/home/

7. Brasil. Ministério da Saúde. DATASUS. Sistema de Informações sobre Mortalidade - SIM, 2007 [texto na Internet]. Brasília; 2007. [citado 2007 set. 20]. Disponível em: www.datasus.gov.br

8. Brasil. Ministério da Saúde. DATASUS. Sistema de Informações sobre Nascidos Vivos - SINASC, 2007 [texto na Internet]. [citado 2007 set. 20]. Disponível em: www.datasus.gov.br

9. Conselho Nacional de Saúde. Resolução n. 196, de 10 de outubro de 1996. Dispõe sobre diretrizes e normas regulamentadoras de pesquisas envolvendo seres humanos. Bioética. 1996;4(2 Supl):15-25
O cuidado de enfermagem é fundamental, ainda, para mobilizar outros profissionais a agirem no tempo adequado, por exemplo, na adoção de protocolo clínico de risco em toda adolescente grávida, na inclusão da família no processo assistencial e na administração de sangue ou em outros suportes no tempo adequado e, por último, na remoção da menina-mulher para centros de referência.

Informações resultantes de trabalhos de pesquisa, com série histórica, fornecem subsídios para que os serviços de saúde e seus profissionais reconheçam o valor de suas práticas e melhorem cada vez mais o que é disponibilizado à população.

10. Yazlle MEHD, Mendes MC, Patta MC, Rocha JSY, Azevedo GD, Marcolin AC. A adolescente grávida: alguns indicadores sociais. Rev Bras Ginecol Obstet. 2002;24(9):609-14.

11. Goldenberg P, Figueiredo MCT, Silva RS. Gravidez na adolescência, pré-natal e resultados perinatais em Montes Claros, Minas Gerais, Brasil. Cad Saúde Pública. 2005;21(4):1077-86.

12. Darroch JE, Singh S, Frost JJ. Differences in teenage pregnancy rates among five developed countries: the roles of sexual activity and contraceptive use. Fam Plann Perspect. 2001;33 (6):244-50, 281. [Erratum in: Fam Plann Perspect. 2002;34 (1):56.

13. Peixoto HCG. A mortalidade materna em Santa Catarina no período de 1996-2002 [texto na Internet]. Florianópolis: Secretaria de Estado da Saúde de Santa Catarina; 2007. [citado 2007 out. 16]. Disponível em: www.saude.sc.gov.br/gestores/ sala_de_leitura/artigos/Mortalidade/Mortalidade \%20Materna _1996-2002.doc

14. Brasil. Lei n. 8.069, de 13 de julho de 1990. Dispõe sobre o Estatuto da Criança e do Adolescente - ECA e dá outras providências. Diário Oficial da União, Brasília, 16 jul.1990.

15. Laurenti R, Jorge MHPM, Gotlieb SLD. A mortalidade materna nas capitais brasileiras: algumas características e estimativa de um fator de ajuste. Rev Bras Epidemiol. 2004;7(4):44960.

16. Gomes FA, Nakano AMS, Almeida AM, Matuo YK. Mortalidade materna na perspectiva do familiar. Rev Esc Enferm USP. 2006;40(1):50-6.

17. Parpinelli MA, Faúndes A, Surita FGC, Pereira BG, Cecatti JG. Mortalidade materna na cidade de Campinas, no período de 1992 a 1994. Rev Bras Ginecol Obstet. 1999;21(4):227-32.

18. Beauvoir S. O segundo sexo: fatos e mitos. Rio de Janeiro: Nova Fronteira; 1949. 